\title{
Malnutrition and Increased Risk of Adverse Outcomes in Elderly Patients Undergoing Elective Colorectal Cancer Surgery: A Case-Control Study Nested in a Cohort
}

\author{
Cristina Martínez-Escribano ${ }^{1,2} \mathbb{D}$, Francisco Arteaga Moreno ${ }^{2} \mathbb{D}$, Marcos Pérez-López ${ }^{2}$, Cristina Cunha-Pérez ${ }^{2}$, \\ Ángel Belenguer-Varea ${ }^{2,3}$, David Cuesta Peredo ${ }^{2,4}{ }^{(\mathbb{D}}$, Francisco Javier Blanco González ${ }^{5}$ and \\ Francisco J. Tarazona-Santabalbina $2,3,6, *$ (D) \\ check for \\ 1 Anesthesiology and Resuscitation, Hospital Universitario de la Ribera, 46600 Valencia, Spain; \\ martinez_criesc@gva.es \\ 2 School of Doctorate, Catholic University of Valencia, San Vicente Martir, 46600 Valencia, Spain; \\ francisco.arteaga@ucv.es (F.A.M.); marcos.perez@mail.ucv.es (M.P.-L.); cristina.cunha@ucv.es (C.C.-P.); \\ belenguer_angvar@gva.es (Á.B.-V.); cuesta_davper@gva.es (D.C.P.) \\ 3 Division of Geriatrics, Hospital Universitario de la Ribera, 46600 Valencia, Spain \\ 4 Quality Management, Hospital Universitario de la Ribera, 46600 Valencia, Spain \\ 5 General and Digestive Surgery, Hospital Universitario de la Ribera, 46600 Valencia, Spain; \\ blanco_fragon@gva.es \\ 6 Centro de Investigación Biomédica en Red Fragilidad y Envejecimiento Saludable (CIBERFES), \\ 28029 Madrid, Spain \\ * Correspondence: tarazona_frasan@gva.es
} updates

Citation: Martínez-Escribano, C.; Arteaga Moreno, F.; Pérez-López, M.; Cunha-Pérez, C.; Belenguer-Varea, Á.; Cuesta Peredo, D.; Blanco González, F.J.; Tarazona-Santabalbina, F.J. Malnutrition and Increased Risk of Adverse Outcomes in Elderly Patients Undergoing Elective Colorectal Cancer Surgery: A Case-Control Study Nested in a Cohort. Nutrients 2022, 14, 207. https://doi.org/10.3390/nu14010207

Academic Editor: Sara Gandini

Received: 26 November 2021 Accepted: 27 December 2021 Published: 3 January 2022

Publisher's Note: MDPI stays neutral with regard to jurisdictional claims in published maps and institutional affiliations.

Copyright: (c) 2022 by the authors Licensee MDPI, Basel, Switzerland. This article is an open access article distributed under the terms and conditions of the Creative Commons Attribution (CC BY) license (https:// creativecommons.org/licenses/by/ $4.0 /)$.

\begin{abstract}
Background: Malnutrition increases worse outcomes during hospital admission for elective colorectal cancer (CRC) surgery in older adults. Methods: This work was designed an observational, monocentric, case-control study nested in a cohort of patients undergoing elective surgery for CRC disease at the Hospital Universitario de la Ribera (HULR) (Alzira, Valencia, Spain) between 2011 and 2019. The study considered patients with a CONUT score in the range of moderate to severe malnutrition ( $>4$ points), with control patients with normal nutritional situations or mild malnutrition. Results: Moderate-to-severe malnutrition cases presented a greater length of stay (LOS), a higher incidence of adverse events (both medical and surgical complications), a higher incidence of surgicalwound infection, a greater need for blood transfusion, and a greater amount of transfused packed red blood cells. During hospitalization, the percentage of patients without nutritional risk decreased from 46 to $9 \%$, and an increase in mild, moderate, and severe risk was observed. Patients with severe nutritional risk at hospital admission had significantly increased mortality at 365 days after discharge (HR: 2.96 (95\% CI 1.14-7.70, $p=0.002)$ ). After adjusting for sex, age, and Charlson index score, patients with severe nutritional risk at admission maintained a higher mortality risk (HR: 3.08 (95\% CI 1.10-8.63, $p=0.032)$ ). Conclusion: Malnutrition prevalence is high in older adults undergoing CRC elective surgery. Furthermore, this prevalence increases during hospital admission. Malnutrition is linked to worse outcomes, such as LOS, surgical and clinical complications, and mortality. For this reason, nutritional interventions are very important in the perioperative period
\end{abstract}

Keywords: colorectal surgery; malnutrition; ERAS; postoperative complications; older patients

\section{Introduction}

Colorectal cancer (CRC) is the third most diagnosed cancer and the second leading cause of death from cancer worldwide [1]. In fact, CRC is the second most prevalent type of cancer in the world, with over 1.4 million cases and 693,900 deaths a year [2]. Age, genetics, and environmental factors, such as obesity, sedentary lifestyle, red meat and processed meat, tobacco and alcohol consumption, diabetes mellitus, and insulin resistance are associated with the development of CRC [3]. Surgery plays an important role, as it 
is the most effective treatment to cure this condition. [4] However, surgery is associated with a high rate of complications, ranging from $8 \%$ to $63 \%$ [5], and an overall perioperative mortality of between $1 \%$ and $12 \%$ [6].

As previously mentioned, advanced age is associated with greater perioperative mortality, a higher rate of perioperative complications, and higher costs [7]. Malnutrition is a powerful predictor of morbidity, mortality, long-term hospitalization, and readmission [8]. Malnutrition can be defined as an unbalanced nutritional state that compromises body reserve and function [9]. Rates of malnutrition in colorectal cancer patients range from $20 \%$ to $37 \%$ [10], depending on the tool used to assess nutritional status. Additionally, malnutrition affects treatment tolerability and postoperative complications, including anastomotic leakage (AL) and oncological outcomes [11].

Controlling nutritional status (CONUT) score [12] is an index that allows for assessment of nutritional condition. CONUT score is calculated from serum albumin, total cholesterol concentration, and peripheral lymphocyte counts. CONUT score is a prognostic factor of postoperative complications [13] and mortality in patients with CRC that useful for estimation of preoperative risk [14]. However, few studies have focused on complications occurring during the perioperative period in patients undergoing elective surgery for CRC. For this reason, our aim was to study the link between nutritional condition assessed with the CONUT tool and perioperative outcomes in patients undergoing elective surgery for CRC disease.

\section{Materials and Methods}

\subsection{Study Design and Subjects}

An observational, monocentric, case-control study nested in a cohort of patients undergoing elective surgery for CRC disease at the Hospital Universitario de la Ribera (HULR) (Alzira, Valencia, Spain) between 2011 and 2019 was designed. HULR is a tertiarycare hospital providing healthcare to a population of 253,330 inhabitants, of which $13.5 \%$ are over 69 years of age.

A patient with a CONUT score in the range of moderate to severe malnutrition (more than 4 points) was considered a case patient, and a patient with a normal nutritional status or mild malnutrition (CONUT equal to or less than 4 points) was considered a control patient.

\subsection{Eligibility Criteria}

Inclusion criteria: patients aged 70 years or older undergoing elective open or laparoscopic colorectal surgery in cancer stage I-III at diagnosis time.

Exclusion criteria: emergency surgery; presence of metastases; patients operated on in other centers and referred to the HULR for sectorization; palliative surgery; life expectancy less than 6 months according to the palliative prognosis score [15].

\subsection{Sample Size}

Data were collected from 371 patients enrolled consecutively, not selected, who underwent elective surgery for CRC between the above-mentioned dates. The calculated power of the study with the sample obtained, with an alpha error of $5 \%$ and a magnitude of effect between groups of $20 \%$, was $97 \%$.

\subsection{Outcome Measures Study Variables}

The following demographic variables were collected: age and sex; anthropometric variables; frailty, measured with criteria established by Balducci [16]; other geriatric syndromes; comorbidities and Charlson index [17]; ASA physical classification status system [18]; tumor staging, blood-test results; and hospital process data, such as admission to the intensive care unit (ICU), length of stay, number of reinterventions, readmissions, and mortality. 
Other variables included were the Fast-track or Enhanced Recovery After Surgery $\left(\right.$ ERAS $\left.^{\circledR}\right)$ protocol assessment in the preoperative period, consisting of anemia management and dietary advice to patients with nutritional deficiency [19]. A rehabilitation specialist conducted respiratory rehabilitation and provided a physical-activity regime tailored to each patient's condition.

The primary outcome was to determine whether a poorer nutritional condition would increase the incidence of complications and adverse events, such as medical (delirium, infections, etc.) and surgical complications (suture dehiscence, paralytic ileus [defined as a lack of transit and oral tolerance established 5 days after surgery]) during hospital admission. Other objectives of the study were to assess the impact of nutritional status at admission and discharge on reinterventions, hospital stay, 30-day readmission rate, and in-hospital and 1-year mortality rate.

\subsection{Statistic Analysis}

The data obtained from the clinical history were analyzed with the statistical software program SPPS, version 23 (SPPS Inc. Chicago, IL, USA). Qualitative variables (including dichotomous variables) were described using absolute and relative frequencies. For quantitative variables, measures of central tendency (mean) were used, along with measures of dispersion (standard deviation, SD). A bivariate calculation was performed for the variables considered in the main and secondary objectives with Student's $t$ test for quantitative variables, with normal distribution, and with the Chi-square technique for qualitative variables. A multivariate analysis was performed using binary logistic regression for the main variable, calculating the crude and adjusted odds ratio (OR). A Cox regression was performed for analysis of mortality at 365 days based on the categorized score of the CONUT score at admission and at hospital discharge, calculating the crude and adjusted hazard ratio (HR). Finally, survival curves were calculated using Kaplan-Meier analysis for the same categories used in the Cox regression. The significance threshold was established at a value of $p<0.05$.

\subsection{Ethical Considerations}

The study complied with legal requirements and good clinical practice guidelines, as well as the Declaration of Helsinki (updated October 2008 version of the World Medical Association on ethical principles for medical research in humans). The protocol was approved by the HULR Ethics and Clinical Research Committee (registry code HULR06112019).

\section{Results}

\section{Subject Characteristics}

A total of 98 patients were considered cases, with 227 controls, according to CONUT score. Cases showed significantly higher comorbidity, as estimated by the Charlson index, with no differences found in age, sex, or anthropometric measures, such as body mass index, tumour stage, ASA score, or frailty. Likewise, the proportion of patients with previous FAST-TRACK or ERAS (enhanced recovery after surgery) evaluation was significantly higher in patients who presented a better nutritional situation on admission (Table 1).

Cases presented a longer hospital stay, a higher incidence of adverse events (both medical and surgical complications), a higher incidence of surgical-wound infections, a greater need for blood transfusion, and a greater amount of transfused packed red blood cells versus control group (Table 2). No statistically significant differences were observed in the incidence of delirium, suture dehiscence, readmission rate, or in-hospital and 365-day mortality.

A binary logistic regression with variables showing statistical significance in the bivariate analysis was performed. Table 3 and shows crude odds ratios and Charlson index ERAS odds ratios (OR) by age and sex with CONUT score a hospital admission and discharge (Table 4), respectively. All variables with significant difference in bivariate analysis maintained significance in the regression analysis. 
Table 1. Bivariate analysis of cases and controls during hospital admission. Baseline situation of cases and controls at hospital admission.

\begin{tabular}{|c|c|c|c|}
\hline Variable. & $\begin{array}{l}\text { Controls } \\
(n=227)\end{array}$ & $\begin{array}{c}\text { Cases } \\
(n=98)\end{array}$ & $p$-Value \\
\hline Age. mean (SD) & $78.1(5.4)$ & $77.4(5.2)$ & 0.204 \\
\hline Gender, $\mathrm{n}(\%)$ & $\begin{array}{l}\sigma^{\top} 123(54 \%) \\
0104(46 \%)\end{array}$ & $\begin{array}{l}\sigma^{\top} 62(63 \%) \\
036(37 \%)\end{array}$ & 0.144 \\
\hline BMI, $\mathrm{Kg} / \mathrm{m}^{2}$ & $\begin{array}{l}28.6(4.5) \\
\text { I } 88(39 \%)\end{array}$ & $\begin{array}{c}28.7(5.1) \\
\text { I } 37(38 \%)\end{array}$ & 0.959 \\
\hline ASA & $\begin{array}{c}\text { II } 131(58 \%) \\
\text { III } 8(3 \%) \\
\text { I } 64(28 \%)\end{array}$ & $\begin{array}{l}\text { II } 58(59 \%) \\
\text { III } 3(3 \%) \\
\text { I } 21(21 \%)\end{array}$ & 0.934 \\
\hline Tumour stage & $\begin{array}{c}\text { II } 72(32 \%) \\
\text { III } 91(40 \%)\end{array}$ & $\begin{array}{l}\text { II } 36(37 \%) \\
\text { III } 41(42 \%)\end{array}$ & 0.261 \\
\hline Charlson Index & $3.1(3.2)$ & $4.3(3.6)$ & 0.006 \\
\hline Frailty & $137(60 \%)$ & $52(63 \%)$ & 0.496 \\
\hline ERAS & $144(63 \%)$ & $46(47 \%)$ & 0.007 \\
\hline
\end{tabular}

Table 2. Results during hospital admission.

\begin{tabular}{cccc}
\hline Variable & $\begin{array}{c}\text { Controls } \\
(\boldsymbol{n}=\mathbf{2 2 7})\end{array}$ & $\begin{array}{c}\text { Cases } \\
(\boldsymbol{n}=\mathbf{9 8})\end{array}$ & $\boldsymbol{p}$-Value \\
\hline Hospital stay & $10.5(7.2)$ & $14.6(14.1)$ & 0.009 \\
Adverse events & $68(32 \%)$ & $42(49 \%)$ & 0.008 \\
Medical complications & $37(16 \%)$ & $30(31 \%)$ & 0.005 \\
Surgical complications & $62(27 \%)$ & $39(40 \%)$ & 0.036 \\
Dehiscence & $4(2 \%)$ & $4(4 \%)$ & 0.249 \\
Surgical-wound infection & $6(3 \%)$ & $10(10 \%)$ & 0.009 \\
Paralytic ileus & $54(24 \%)$ & $33(34 \%)$ & 0.076 \\
Delirium & $18(8 \%)$ & $8(8 \%)$ & 0.943 \\
Transfusion & $58(26 \%)$ & $42(43 \%)$ & 0.003 \\
Number of concentrates & $0.8(2.0)$ & $1.5(2.4)$ & 0.012 \\
Readmissions & $133(59 \%)$ & $67(68 \%)$ & 0.107 \\
Reinterventions & $19(8 \%)$ & $8(7 \%)$ & 0.457 \\
ICU admission & $6(3 \%)$ & $6(6 \%)$ & 0.195 \\
In-patient mortality & $9(4 \%)$ & $3(3 \%)$ & 0.939 \\
365-day mortality & $20(9 \%)$ & $12(12 \%)$ & 0.417
\end{tabular}

Legend: BMI: body mass index; ASA: American Society Anesthesiology scale; ERAS: Enhanced Recovery After Surgery program.

Table 3. Univariate analysis variables associated with nutritional impairment estimated by CONUT score. Crude odds ratio and Charlson Comorbidity Index and ERAS odds ratios adjusted by age and sex. Univariate analysis variables associated with nutritional impairment estimated by CONUT score at hospital admission.

\begin{tabular}{ccccc}
\hline & $\begin{array}{c}\text { Crude OR } \\
\mathbf{( 9 5 \%} \mathbf{C I})\end{array}$ & $\boldsymbol{p}$-Value & $\begin{array}{c}\text { Adjusted OR } \\
\mathbf{( 9 5 \%} \mathbf{~ C I})\end{array}$ & $p$-Value \\
\hline Paralytic ileus & $0.685(0.414-1.131)$ & 0.139 & $0.732(0.435-1.233)$ & 0.245 \\
Adverse events & $2.021(1.212-3.372)$ & 0.007 & $1.247(1.127-3.333)$ & 0.017 \\
Surgery wound infection & $4.186(1.477-11.864)$ & 0.007 & $5.780(1.851-18.049)$ & 0.003 \\
Length of stay >12 days & $2.004(1.197-3.356)$ & 0.008 & $1.184(1.064-3.187)$ & 0.029 \\
In-hospital mortality & $0.693(0.203-2.888)$ & 0.693 & $0.848(0.217-3.314)$ & 0.848 \\
Transfusion & $2.185(1.327-3.600)$ & 0.002 & $2.127(1.221-3.704)$ & 0.008 \\
Medical complications & $2.266(1.300-3.948)$ & 0.004 & $2.688(1.475-4.897)$ & 0.001 \\
Surgical complications & $1.759(1.068-2.897)$ & 0.026 & $2.317(1.394-3.849)$ & 0.001 \\
\hline
\end{tabular}


Table 4. Univariate analysis variables associated with nutritional impairment estimated by CONUT score at hospital discharge.

\begin{tabular}{ccccc}
\hline & $\begin{array}{c}\text { Crude OR } \\
\mathbf{( 9 5 \% ~ C I ) ~}\end{array}$ & $p$-Value & $\begin{array}{c}\text { Adjusted OR } \\
\mathbf{( 9 5 \% ~ C I ) ~}\end{array}$ & $p$-Value \\
\hline Paralytic ileus & $2.444(1.463-4.084)$ & 0.001 & $2.478(1.457-4.214)$ & 0.001 \\
Adverse events & $2.595(1.600-4.209)$ & $<0.001$ & $2.673(1.609-4.442)$ & $<0.001$ \\
Surgery-wound infection & $0.968(0.355-2.645)$ & 0.950 & $0.933(0.324-2.690)$ & 0.898 \\
Urinary tract infection & $9.172(1.149-73.247)$ & 0.037 & $9.421(1.095-81.04)$ & 0.041 \\
Length of stay >12 days & $2.135(1.286-3.544)$ & 0.003 & $1.956(1.155-3.313)$ & 0.013 \\
In-hospital mortality & $12.468(1.602-97.036)$ & 0.016 & $14.668(1.853-116.1)$ & 0.011 \\
Transfusion & $3.573(2.155-5.923)$ & $<0.001$ & $3.002(1.751-5.146)$ & $<0.001$ \\
Medical complications & $2.633(1.490-4.654)$ & 0.001 & $2.730(1.499-4.974)$ & 0.001 \\
Surgical complications & $2.235(1.377-3.627)$ & 0.001 & $2.220(1.340-3.680)$ & 0.002 \\
\hline
\end{tabular}

During hospitalization. the percentage of patients with nutritional risk increased in all groups-mild. moderate. and sever-overall. from 9 to $46 \%$. Therefore. mild nutritional impairment estimated by CONUT increased from 25 to $38 \%$. moderate nutritional impairment increased from 21 to $40 \%$. and severe nutritional impairment increased from 8 to $13 \%$. $p<0.001$ (Figure 1).
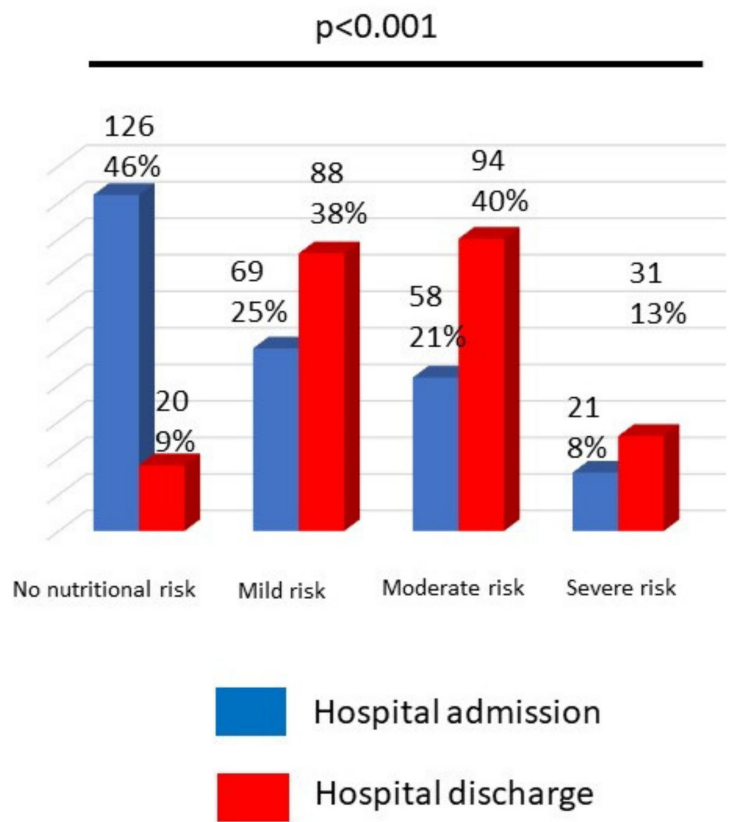

Figure 1. Changes in nutritional risk estimated by CONUT score.

Bivariate CONUT score calculation was repeated with hospital discharge blood-test results. Patients with nutritional impairment at hospital discharge presented a higher incidence of paralytic ileus and urinary tract infection and a higher rate of readmission in intensive care units (ICU) (Table 5). Similarly to the previous analysis. when repeating the analysis using a logistic regression. variables maintained statistical significance. 
Table 5. Bivariate analysis with nutritional status at hospital admission.

\begin{tabular}{cccc}
\hline Variable & $\begin{array}{c}\text { CONUT without } \\
\text { Nutritional Disorder } \\
(\boldsymbol{n}=\mathbf{1 6 0 )}\end{array}$ & $\begin{array}{c}\text { CONUT with } \\
\text { Nutritional Disorder } \\
(\boldsymbol{n}=\mathbf{1 6 7 )}\end{array}$ & $p$-Value \\
\hline Mortality & $0(0 \%)$ & $13(8 \%)$ & $<0.001$ \\
Mortality 365 days & $7(4 \%)$ & $26(16 \%)$ & 0.001 \\
Adverse events & $40(27 \%)$ & $72(48 \%)$ & $<0.001$ \\
Number of adverse events & $1.1(2.4)$ & $2.1(3.4)$ & 0.003 \\
Reintervention & $9(6 \%)$ & $19(11 \%)$ & 0.076 \\
Paralytic ileus & $29(18 \%)$ & $58(35 \%)$ & 0.001 \\
Suture dehiscence & $4(3 \%)$ & $10(6 \%)$ & 0.172 \\
ICU readmissons & $2(1 \%)$ & $11(7 \%)$ & 0.020 \\
Urinary tract infection & $1(1 \%)$ & $9(5 \%)$ & 0.020 \\
Surgery-wound infection & $8(5 \%)$ & $8(5 \%)$ & 1.000 \\
Transfusion & $28(18 \%)$ & $74(44 \%)$ & $<0.001$ \\
Number of concentrates & $0.4(1.1)$ & $1.6(2.7)$ & $<0.001$ \\
Medical complications & $21(13 \%)$ & $47(28 \%)$ & 0.001 \\
Surgical complications & $35(22 \%)$ & $66(40 \%)$ & 0.001 \\
ERAS & $105(66 \%)$ & $86(51 \%)$ & 0.010 \\
Frailty & $93(58 \%)$ & $108(65 \%)$ & 0.256 \\
Delirium & $10(6 \%)$ & $16(10 \%)$ & 0.310 \\
\hline
\end{tabular}

Patients with severe nutritional risk at hospital admission presented a statistically significant increase in mortality at 365 days (HR: 2.96 (95\% CI 1.14-7.70. $p=0.002)$ ). but this significance was not observed in patients with severe nutritional deficit at hospital discharge (HR: 5.24 (95\% CI 0.64-42.6. $p=0.053)$ ). After adjusting for sex. age. and Charlson index score. patients with severe nutritional risk at admission maintained statistical significance (HR: 3.08 (95\% CI 1.10-8.63. $p=0.032)$ ) with the most severe nutritional risk at hospital discharge reaching HR significance: 4.03 (95\% CI 0.49-33.3. $p=0.195)$. Similarly. Tables 6 and 7 and Figure 2A,B show the lower overall survival of patients with severe nutritional risk at hospital admission $(p=0.001)$ and at hospital discharge $(p=0.031)$.

Table 6. Survival mean (days) using the Kaplan-Meier analysis based on nutritional status estimated by CONUT. Analysis with the nutritional estimate using CONUT at hospital admission.

\begin{tabular}{cccc}
\hline \multirow{2}{*}{ Mean Estimation } & \multicolumn{2}{c}{$\mathbf{9 5 \% \text { Confidence Interval }}$} \\
\cline { 3 - 4 } & & Lower Limit & Upper Limit \\
\hline No nutritional risk & 341.437 & 327.837 & 355.036 \\
Mild risk & 359.870 & 349.887 & 369.852 \\
Moderate risk & 350.241 & 335.044 & 365.439 \\
Severe risk & 285.524 & 228.997 & 342.050 \\
\hline
\end{tabular}

Table 7. Analysis with the nutritional estimate using CONUT at hospital discharge.

\begin{tabular}{cccc}
\hline \multirow{2}{*}{ Mean Estimation } & \multicolumn{2}{c}{$\mathbf{9 5 \% \text { Confidence Interval }}$} \\
\cline { 3 - 4 } & & Lower Limit & Upper Limit \\
\hline No nutritional risk & 354.900 & 335.605 & 374.195 \\
Mild risk & 355.682 & 346.066 & 365.298 \\
Moderate risk & 338.787 & 322.305 & 355.270 \\
Severe risk & 297.806 & 249.505 & 346.108 \\
\hline
\end{tabular}


A)

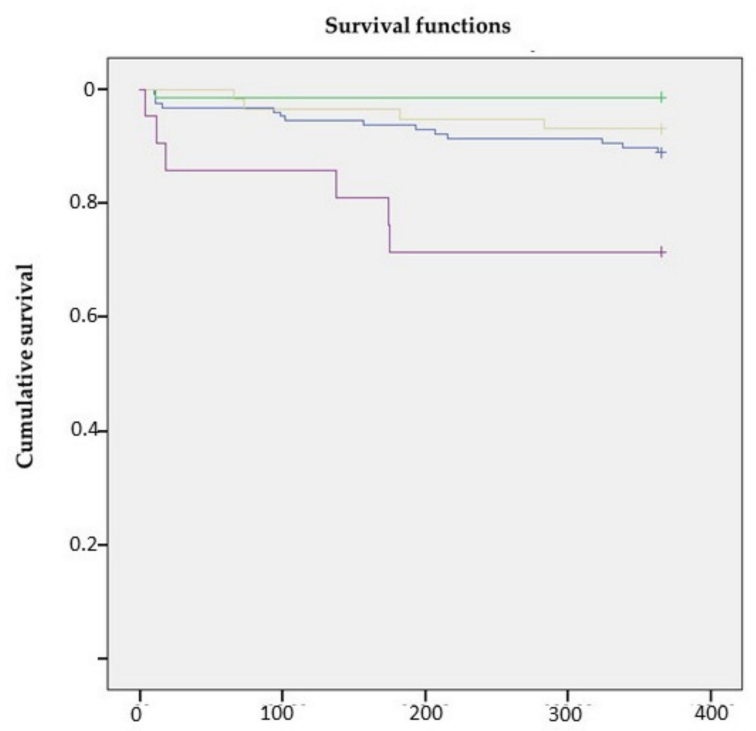

CONUT 4 initial categorie

$=$ No nutritional risk

Moderate nutritional risk
Severe nutritiontion

Severe nutritional risk
+ No nutritional risk-censored
Mild nutritional risk-censored

Moderate nutritional risk-censor

B)

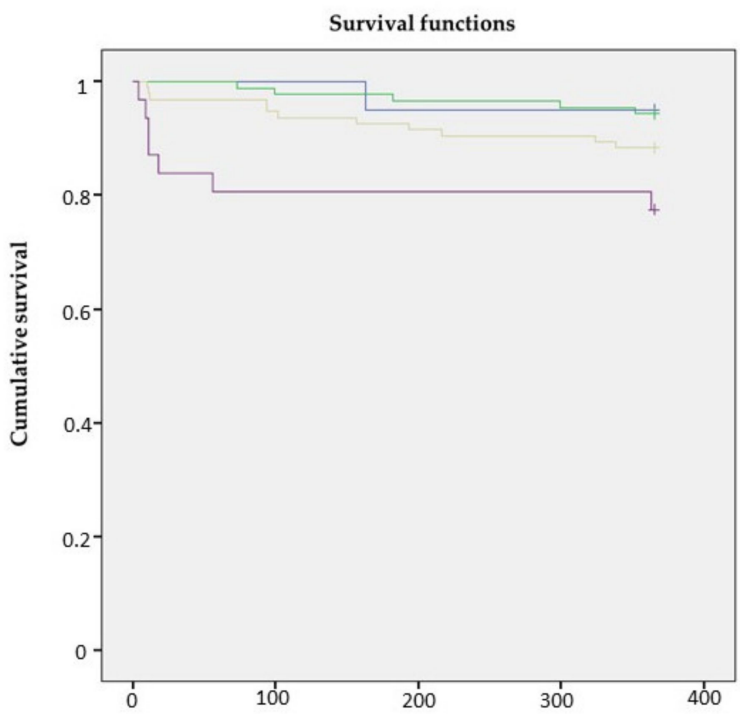

Conut $\mathbf{4}$ final categories

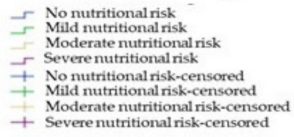

Figure 2. Survival curve using the Kaplan-Meier analysis based on the nutritional status estimated by CONUT. (A) Survival curve with the nutritional estimate using CONUT at hospital admission. (B) Analysis with the nutritional estimate using CONUT at hospital discharge.

\section{Discussion}

In our study. the prevalence of malnutrition estimated by CONUT score was high in older adults undergoing elective surgery for CRC. This prevalence increased significantly during hospital admission. Malnutrition during hospital admission was associated with a longer hospital stay and a higher incidence of adverse events. intensive care unit readmissions. and mortality.

CONUT is a simple and useful nutritional screening tool that has previously been used to estimate the nutritional status of patients undergoing elective surgery for CRC [12,13]. CONUT score has been associated with lower survival $[13,20,21]$ and postoperative complications [12] in patients undergoing elective surgery for colorectal neoplasia.

However. few studies have focused on complications occurring during the perioperative period in patients undergoing elective surgery for CRC. In our study. we observed a significant increase in the prevalence of malnutrition from hospital admission to discharge. Preoperative CONUT score has previously been correlated with a higher mortality rate [22]. 
although not with increased length of stay. transfusion requirements. surgical-wound and urinary tract infections. or ICU readmissions observed in our study. Surgical-wound infection was associated with CONUT score at admission but not at hospital discharge. A recent study found that surgical-wound infection was linked to non-administration of preoperative enteral nutrition [23]. Nutritional intervention included in the ERAS program is crucial for the reduction of adverse events. such as surgical-wound infection.

Previous studies have reported that poorer nutritional condition is associated with a longer hospital stay and a higher incidence of wound-suture dehiscence [24]. as well as paralytic ileus [25]. However. CONUT has not been used as a nutritional screening tool. In our study. a higher incidence of paralytic ileus was observed with the CONUT score at hospital discharge but not at admission time. Regarding the study design. we were not able to report on whether the association between the CONUT score at hospital discharge and paralytic ileus is a cause or consequence. On the other hand. a statistically significant increased incidence of suture dehiscence was not observed. Patients with a CONUT score in the range of malnutrition had an increased risk of needing red-blood-cell transfusion. This relationship between poorer nutritional condition and a higher presence of anemia has been previously described [26] but not using CONUT as a nutritional screening tool.

Two variables influenced the nutritional status of older adults undergoing elective surgery for CRC: one negatively-comorbidity estimated by the Charlson index; the second. positively-prior assessment and intervention within the ERAS program. The Charlson index has been associated with poorer nutritional status [27]. while the ERAS program has been shown to improve the nutritional status of patients undergoing this surgery [28].

Despite the nutritional improvement described for the ERAS. we observed a significant deterioration in nutritional status during hospital admission. After less than 15 days of length of hospital stay. we found a very significant decrease in the percentage of patients without nutritional risk estimated by CONUT. Previous poor nutritional status has been previously described as a risk factor for bad outcomes during hospital admission for elective colorectal cancer surgery $[8,29,30]$. However. nutritional deterioration during hospital admission for elective colorectal cancer surgery had not been previously reported. These results suggest the need to intensify nutritional intervention during hospital admission.

The main limitations of the study are the long recruitment period (8 years). the greater comorbidity of the cases. estimated by means of the ICC. and the greater number of patients with ERAS intervention in the controls. For this reason. both variables have been used as adjustment variables in the logistic regression analysis. The percentage of lost data was calculated as $12 \%$.

\section{Conclusions}

A poorer nutritional condition is correlated with a longer hospital stay. a higher incidence of complications. and a higher mortality. Nutritional condition worsens during the hospitalization of these patients and. compared to the nutritional situation on admission. is associated with a higher incidence of paralytic ileus and urinary tract infection. CONUT score is a useful nutritional screening tool in patients undergoing CRC elective surgery and can help to assess the nutritional evolution during hospital admission due to elective colorectal cancer surgery. ERAS could also reduce the prevalence of nutritional impairment in hospital admission. and these results suggest that intensification of this program before and during hospitalization could improve the nutritional status of these patients and reduce the incidence of complications and mortality rate.

Author Contributions: Conceptualization. C.M.-E., M.P.-L. and F.J.T.-S.; methodology. C.M.-E., F.A.M., M.P.-L.; software. M.P.-L. and F.J.T.-S.; validation. C.M.-E. and M.P.-L.; formal analysis. C.M.-E. and F.J.T.-S.; investigation. C.M.-E. and M.P.-L.; resources. D.C.P. and F.J.B.G.; data curation. C.M.-E. and Á.B.-V.; writing-original draft preparation. C.C.-P. and F.J.T.-S.; writing-review and editing. F.J.T.-S.; visualization. M.P.-L.; supervision. F.J.T.-S.; project administration. D.C.P.; funding acquisition. C.M.-E. All authors have read and agreed to the published version of the manuscript. 
Funding: This research received no external funding.

Institutional Review Board Statement: The study was conducted according to the guidelines of the Declaration of Helsinki and approved by the Institutional Ethics Committee of Hospital Universitario de La Ribera (Alzira. Valencia. Spain) with approval number HULR1327; date of approval. 14 June 2017.

Informed Consent Statement: Informed consent was obtained from all subjects involved in the study.

Data Availability Statement: The study did not report any data.

Conflicts of Interest: The authors declare no conflict of interest.

\section{References}

1. Rawla, P.; Sunkara, T.; Barsouk, A. Epidemiology of colorectal cancer: Incidence, mortality, survival, and risk factors. Prz Gastroenterol. 2019, 14, 89-103. [CrossRef]

2. Torre, L.A.; Bray, F.; Siegel, R.L.; Ferlay, J.; Lortet-Tieulent, J.; Jemal, A. Global Cancer statistics, 2012. CA Cancer J. Clin. 2015, 65, 87-108. [CrossRef]

3. Thanikachalam, K.; Khan, G. Colorectal Cancer and Nutrition. Nutrients 2019, 11, 164. [CrossRef]

4. Pallis, A.G.; Papamichael, D.; Audisio, R.; Peeters, M.; Folprecht, G.; Lacombe, D.; Van Cutsem, E. EORTC Elderly Task Force experts' opinion for the treatment of colon cancer in older patients. Cancer Treat. Rev. 2010, 36, 83-90. [CrossRef]

5. Fagard, K.; Wolthuis, A.; Verhaegen, M.; Flamaing, J.; Deschodt, M.A. retrospective observational study of enhanced recovery after surgery in older patients undergoing elective colorectal surgery. PLoS ONE 2020, 15, e0232857. [CrossRef]

6. Okabe, H.; Ohsaki, T.; Ogawa, K.; Ozaki, N.; Hayashi, H.; Akahoshi, S.; Ikuta, Y.; Ogata, K.; Baba, H.; Takamori, H. Frailty predicts severe postoperative complications after elective colorectal surgery. Am. J. Surg. 2019, 217, 677-681. [CrossRef] [PubMed]

7. Bowel Cancer Incidence Statistics. Available online: https:/ /www.cancerresearchuk.org/health-professional/cancer-statistics/ statistics-by-cancer-type/bowel-cancer/incidence (accessed on 20 December 2021).

8. Seretis, C.; Kaisari, P.; Wanigasooriya, K.; Shariff, U.; Youssef, H. Malnutrition is associated with adverse postoperative outcome in patients undergoing elective colorectal cancer resections. J BUON 2018, 23, 36-41. [PubMed]

9. Gillis, C.; Wischmeyer, P.E. Pre-operative nutrition and the elective surgical patient: Why, how and what? Anaesthesia 2019, 74 (Suppl. S1), 27-35. [CrossRef] [PubMed]

10. Burden, S.T.; Hill, J.; Shaffer, J.L.; Todd, C. Nutritional status of preoperative colorectal cancer patients. J. Hum. Nutr. Diet. 2010, 23, 402-407. [CrossRef] [PubMed]

11. Yamano, T.; Yoshimura, M.; Kobayashi, M.; Beppu, N.; Hamanaka, M.; Babaya, A.; Tsukamoto, K.; Noda, M.; Matsubara, N.; Tomita, N. Malnutrition in rectal cancer patients receiving preoperative chemoradiotherapy is common and associated with treatment tolerability and anastomotic leakage. Int. J. Colorectal Dis. 2016, 31, 877-884. [CrossRef]

12. Ignacio de Ulíbarri, J.; González-Madroño, A.; de Villar, N.G.; González, P.; González, B.; Mancha, A.; Rodríguez, F.; Fernández, G. CONUT: A tool for controlling nutritional status. First Valid. A Hosp. Popul. Nutr. Hosp. 2005, 20, $38-45$.

13. Ahiko, Y.; Shida, D.; Horie, T.; Tanabe, T.; Takamizawa, Y.; Sakamoto, R.; Moritani, K.; Tsukamoto, S.; Kanemitsu, Y. Controlling nutritional status (CONUT) score as a preoperative risk assessment index for older patients with colorectal cancer. BMC Cancer 2019, 19, 946. [CrossRef]

14. Tokunaga, R.; Sakamoto, Y.; Nakagawa, S.; Ohuchi, M.; Izumi, D.; Kosumi, K.; Taki, K.; Higashi, T.; Miyamoto, Y.; Yoshida, N.; et al. Conut: A novel independent predictive score for colorectal cancer patients undergoing potentially curative resection. Int. $J$. Color. Dis. 2017, 32, 99-106. [CrossRef]

15. Iseki, Y.; Shibutani, M.; Maeda, K.; Nagahara, H.; Ohtani, H.; SMorita, T.; Tsunoda, J.; Inoue, S.; Chihara, S. The Palliative Prognostic Index: A scoring system for survival prediction of terminally ill cancer patients. Support. Care Cancer 1999, 7, 128-133. [CrossRef]

16. Balducci, L.; Colloca, G.; Cesari, M.; Gambassi, G. Assessment and treatment of elderly patients with cancer. Surg. Oncol. 2010, 19, 117-123. [CrossRef] [PubMed]

17. Charlson, M.E.; Pompei, P.; Ales, K.L.; MacKenzie, C.R. A new method of classifying prognostic comorbidity in longitudinal studies: Development and validation. J. Chronic Dis. 1987, 40, 373-383. [CrossRef]

18. Saklad, M. Grading of patients for surgical procedures. Anesthesia 1941, 2, 281-284. [CrossRef]

19. Kehlet, H.; Wilmore, D.W. Multimodal strategies to improve surgical outcome. Am. J. Surg. 2002, 183, 630-641. [CrossRef]

20. Takagi, K.; Buettner, S.; Ijzermans, J.N.M. Prognostic significance of the controlling nutritional status (CONUT) score in patients with colorectal cancer: A systematic review and meta-analysis. Int. J. Surg. 2020, 78, 91-96. [CrossRef]

21. Hayama, T.; Ozawa, T.; Okada, Y.; Tsukamoto, M.; Fukushima, Y.; Shimada, R.; Nozawa, K.; Matsuda, K.; Fujii, S.; Hashiguchi, Y. The pretreatment Controlling Nutritional Status (CONUT) score is an independent prognostic factor in patients undergoing resection for colorectal cancer. Sci. Rep. 2020, 10, 13239. [CrossRef]

22. Hiramatsu, K.; Shindoh, J.; Hanaoka, Y.; Toda, S.; Ueno, M.; Matoba, S.; Kuroyanagi, H. Postoperative Nutritional Status is Predictive of the Survival Outcomes in Patients Undergoing Resection of Stage III Colorectal Cancer. World J. Surg. 2021, 45, 3198-3205. [CrossRef] 
23. Páramo-Zunzunegui, J.; Alonso-García, M.; Rodríguez-Villar, D.; Drewniak-Jakubowska, J.; Calvo-Espino, P.; Cuberes-Montserrat, R.; Rodríguez-Caravaca, G.; Durán-Poveda, M. Incidence of surgical infection and risk factors in colorectal surgery-A prospective cohort study. Cir. Cir. 2021, 89, 156-162.

24. Karin, M.; Bogut, A.; Hojsak, I.; Babić, E.; Volarić, M.; Bevanda, M. Nutritional status and its effect on complications in patients with colorectal cancer. Wien. Klin Wochenschr. 2020, 132, 431-437. [CrossRef]

25. Barreiro Domínguez, E.; Sánchez Santos, R.; Diz Jueguen, S.; Piñeiro Teijeiro, A.; Seoane Antelo, J.; Carrera Dacosta, E. Impacto de la terapia con inmunonutrición oral perioperatoria en pacientes sometidos a cirugía por cáncer colorrectal [Impact of preoperative oral nutrition therapy in patients undergoing surgery for colorectal cancer]. Nutr. Hosp. 2019, 36, 1150-1156. [PubMed]

26. Hardy, P.Y.; Degesve, M.; Joris, J.; Coimbra, C.; Decker, E.; Hans, G. Impact of Preoperative Anemia on Outcomes of Enhanced Recovery Program after Colorectal Surgery: A Monocentric Retrospective Study. World J. Surg. 2021, 45, 2326-2336. [CrossRef]

27. Cooper, L.; Siam, B.; Sagee, A.; Orgad, R.; Levi, Y.; Wasserberg, N.; Beloosesky, Y.; Kashtan, H. Some Nursing Screening Tools Can be Used to Assess High-Risk Older Adults Who Undergo Colorectal Surgery for Cancer. Clin. Interv. Aging. 2020, 15, 1505-1511. [CrossRef]

28. Yeung, S.E.; Hilkewich, L.; Gillis, C.; Heine, J.A.; Fenton, T.R. Protein intakes are associated with reduced length of stay: A comparison between Enhanced Recovery after Surgery (ERAS) and conventional care after elective colorectal surgery. Am. J. Clin. Nutr. 2017, 106, 44-51.

29. Chiang, J.M.; Chang, C.J.; Jiang, S.F.; Yeh, C.Y.; You, J.F.; Hsieh, P.S.; Huang, H.Y. Pre-operative serum albumin level substantially predicts post-operative morbidity and mortality among patients with colorectal cancer who undergo elective colectomy. Eur J. Cancer Care 2017, 26, e12403. [CrossRef] [PubMed]

30. Tarazona-Santabalbina, F.J.; Llabata-Broseta, J.; Belenguer-Varea, Á.; Álvarez-Martínez, D.; Cuesta-Peredo, D.; Avellana-Zaragoza, J.A. A daily multidisciplinary assessment of older adults undergoing elective colorectal cancer surgery is associated with reduced delirium and geriatric syndromes. J. Geriatr. Oncol. 2019, 10, 298-303. [CrossRef] [PubMed] 